\title{
Multiple Glucagon-Producing Pancreatic Neuroendocrine Tumors in a Horse (Equus caballus)
}

Veterinary Pathology

20I4, Vol. 5I(3) 607-6II

(C) The Author(s) 2013

Reprints and permission:

sagepub.com/journalsPermissions.nav DOI: I0.I I77/03009858/3492803

vet.sagepub.com

(S)

\author{
N. Herbach', L. Nagel', T. Zwick ${ }^{2}$, and W. Hermanns'
}

\begin{abstract}
Pancreatic neuroendocrine tumors of glucagon-producing cells are extremely rare in domestic animals. In this report, we describe for the first time, to our knowledge, the incidental finding of multiple glucagon-producing neuroendocrine tumors of the pancreas of a horse. The animal was euthanized due to severe local infection after tooth extraction. On postmortem examination, multiple white nodules of up to $4 \mathrm{~cm}$ in diameter were observed in the pancreas. Histologically, pancreatic nodules had the appearance of neuroendocrine neoplasms with positive immunoreactivity for glucagon, synaptophysin, chromogranin $A$, and neuron-specific enolase. Electron microscopy revealed numerous electron-dense granules, similar to those observed in normal pancreatic alpha cells, in the neoplastic cells. In addition, the left adrenal gland showed multiple hyperplastic foci and adenomas in the medulla that were identified as pheochromocytomas. Based on the morphologic appearance and immunohistochemical staining pattern of pancreatic nodules, a diagnosis of multiple glucagon-producing neuroendocrine tumors was made.
\end{abstract}

\section{Keywords}

glucagonoma, pancreatic neuroendocrine tumor, multiple islet-cell adenomas, horse

The neuroendocrine system can be divided into the glandforming and the diffuse/disseminated neuroendocrine system (DNES). The gland-forming group includes the pituitary pars distalis, parathyroid, paraganglia, and adrenal medulla. Cell types of the DNES may be found in the pancreas, thyroid, skin, lung, and thymus, as well as in the bilary, urogenital, and gastrointestinal tracts. $^{6}$

Pancreatic neuroendocrine neoplasms (Pan-NENs) in humans and domestic animals can be divided into 2 groups: functioning and nonfunctioning neoplasms. Functioning tumors produce and secrete hormones, thereby inducing hormonal syndromes (eg, insulinomas, glucagonomas, gastrinomas, vasoactive intestinal peptide [VIP]-omas). ${ }^{5,7}$ Insulinomas are frequently observed in older dogs, adult ferrets, and rarely in cats. These neoplasms are usually multihormonal, containing cells expressing somatostatin, glucagon, or pancreatic polypeptide. ${ }^{8,11}$ Glucagonomas are rare in domestic animals, occurring less frequently than nonfunctional Pan-NENs and insulinomas in humans. ${ }^{2,5}$ These tumors can be associated with diabetes mellitus and superficial necrolytic dermatitis or necrolytic migratory erythema. ${ }^{1,3}$ Gastrinomas cause Zollinger-Ellison syndrome in dogs and humans, but most gastrinomas are of duodenal origin, since gastrin-producing cells do not exist in the adult canine or human pancreas. ${ }^{5}$ VIP-omas are rare in both animals and humans and can cause diarrhea as part of the Verner-Morrison syndrome in humans. ${ }^{5}$
Nonfunctioning pancreatic neuroendocrine tumors produce hormones but do not exhibit appreciable hormonal secretion or hormone-related syndromes. ${ }^{5,7}$ Most nonfunctioning tumors in animals are composed of PP, alpha, or delta cells. ${ }^{5}$ Nonfunctioning tumors that are composed of cells that exhibit immunoreactivity for glucagon, for example, are called glucagon-producing NENs. ${ }^{7}$

According to the latest human World Health Organization (WHO) classification, tumors of the gastroenteropancreatic neuroendocrine system are generally called neuroendocrine neoplasms (NENs). NENs are divided into the welldifferentiated neuroendocrine tumors (NETs), which are further subdivided into grade (G) 1 and G2 tumors, and poorly differentiated neuroendocrine carcinomas (NECs), which are considered G3 tumors. Tumor grades 1 to 3 increase in order of increasing proliferative activity. ${ }^{7}$ Islet-cell tumors in

\footnotetext{
'Institute of Veterinary Pathology, Center for Clinical Veterinary Medicine, Ludwig-Maximilians-University, Munich, Germany

${ }^{2}$ Veterinary Clinic Gessertshausen, Gessertshausen, Germany

Corresponding Author:

N. Herbach, Institute of Veterinary Pathology, Center for Clinical Veterinary Medicine, Ludwig-Maximilians-University, Veterinaerstr. 13, 80539 Munich, Germany.

Email: n.herbach@Imu.de
} 
veterinary medicine are classified as adenomas or carcinomas. The benign adenomas tend to appear solitary in dogs and cats and multiple in ferrets and cattle. Carcinomas are malignant neoplasms that are characterized by infiltration into surrounding tissue, blood vessels, and/or lymphatics. They may metastasize to regional lymph nodes or to the liver and show a high proliferative index as well as areas of hemorrhage and necrosis. $^{5}$

The present study describes the incidental finding of multiple pancreatic glucagon-producing neuroendocrine neoplasms/ adenomas, which, to our knowledge, has not been previously reported in the horse.

Immunohistochemistry was performed on paraffinembedded tissue sections. Antigen retrieval was performed using proteinase $\mathrm{K}$ (synaptophysin) or microwave pretreatment with citrate buffer ( $\mathrm{pH} \mathrm{6.0)}$ for 20 minutes (chromogranin A). Primary antibodies used for immunohistochemistry included rabbit anti-chromogranin A (1:500 in phosphate-buffered saline [PBS], pH 7.4; Dako Deutschland GmbH, Hamburg, Germany), rabbit anti-neuron-specific enolase (1:500 in PBS; Biomol GmbH, Hamburg, Germany), rabbit anti-synaptophysin (1:100 in PBS; Dako), guinea pig anti-porcine insulin (1:2000 in PBS; Dako), rabbit anti-human glucagon (1:500 in PBS; Dako), rabbit anti-human somatostatin (1:300 in PBS; Dako), or rabbit anti-human pancreatic polypeptide (1:700 in PBS; Dako), respectively. A biotinylated secondary antibody was used to detect antibodies against chromogranin A, neuron-specific enolase, and synaptophysin, using the avidin-biotin complex method (VECTOR Laboratories Ltd, Peterborough, UK), and a peroxidase-conjugated secondary antibody was used to detect antibodies against insulin, glucagon, somatostatin, and PP (Dako); DAB served as chromogen.

Samples for electron microscopy were postfixed in $1 \%$ osmium tetroxid and routinely embedded in Epon. Ultrathin sections $(70-80 \mathrm{~nm})$ were stained with uranyl citrate and lead citrate and examined with a transmission electron microscope (EM10; Zeiss, Oberkochen, Germany).

The 23-year-old Württemberger crossbred gelding was euthanized due to severe local infection following tooth extraction. Postmortem examination revealed severe necrotizing inflammation of the alveolar bone surrounding the extracted tooth as well as adjacent skin, muscles, and connective tissue of the head, the frontal, maxillary, and conchal sinuses, cranial nerves, pituitary and pineal glands; and the ventral meninges. Microbiological examination revealed a mixed flora consisting of Staphylococcus aureus, Escherichia coli, Candida spp, Aspergillus fumigatus, and Mucoraceae in the alveole, sinuses, and the meninges. In addition, bacteria-containing thrombi were observed in histological sections of the lungs, indicating hematogenous spread.

The pancreas contained multiple, well-circumscribed, round nodules measuring 0.2 to $4 \mathrm{~cm}$ in diameter that were white on section with focal hemorrhage and necrosis in larger masses (Fig. 1). Histologically, the masses were identified as neoplasms surrounded by a fibrous capsule, a trabecular or glandular arrangement of neoplastic cells, and only occasional

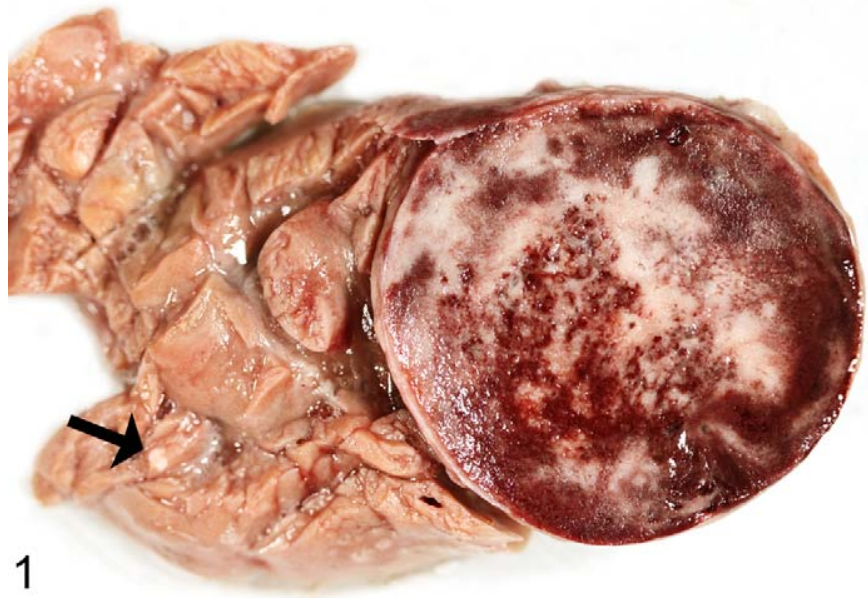

Figure I. Pancreas; horse. Pancreatic tumor of $4 \mathrm{~cm}$ in diameter, showing a white cross section, multifocal areas of hemorrhage, and necrosis; the arrow indicates a microadenoma of $2 \mathrm{~mm}$ in diameter.

Zellballen formation (Fig. 3). The tumor cells were well differentiated and uniform, showing a lightly eosinophilic to amphophilic granular cytoplasm, round to oval nuclei and inconspicuous nucleoli (Figs. 2-4). The mitotic index was low $\left(<10\right.$ per $\left.2 \mathrm{~mm}^{2}\right)$. In some tumors, multifocal hemorrhage and necrosis corresponded to grossly observed cystic areas.

Neoplastic cells showed moderate diffuse cytoplasmic immunoreactivity for glucagon (Fig. 5), the neuroendocrine markers synaptophysin (Fig. 6) and chromogranin A, and strong diffuse cytoplasmic immunoreactivity for neuronspecific enolase (not shown). Tumor cells were negative for insulin (Fig. 7), somatostatin, and PP (not shown).

Besides glucagon-producing tumors, multifocal areas of alpha-cell hyperplasia could be detected in the pancreas by immunohistochemistry, and in addition, physiologically sized islets also contained numerous alpha cells (Fig. 5), apart from numerous insulin-producing cells and very few delta and PP cells (not shown).

Electron microscopically, numerous electron-dense granules measuring about 230 to $400 \mathrm{~nm}$ in diameter could be detected in the tumor cells, similar to secretory granules commonly seen in alpha cells associated with glucagon storage (Fig. 8).

According to histological, immunohistochemical, and electron microscopic findings and due to the lack of a clinically overt hormonal syndrome, the diagnosis of multiple glucagonproducing NETs (human WHO) or nonfunctional alpha-cell adenomas (veterinarian WHO classification) was made.

In addition, multiple red or beige tumors were observed in the medulla of the left adrenal gland. Histology and immunohistochemistry revealed multifocal hyperplasia of the adrenal medulla and multiple pheochromocytomas (not shown).

Nonfunctioning Pan-NENs may produce large amounts of hormones but, by definition, are considered nonfunctional because these hormones are not secreted or do not produce a clinical syndrome. In the presented case, a nonfunctioning 

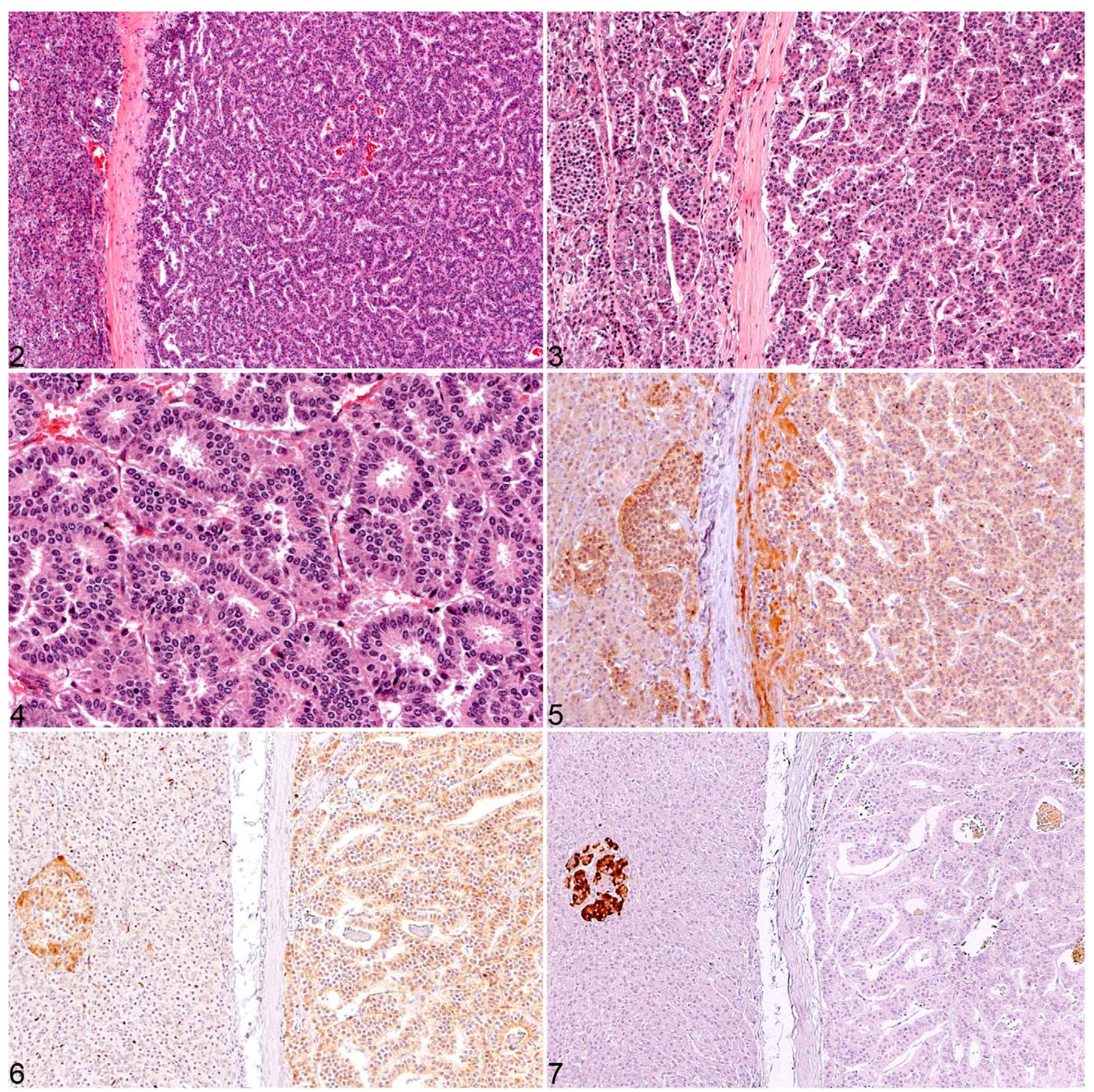

Figure 2. Pancreas; horse. Islet-cell tumor showing a fibrous capsule, with well-differentiated tumor cells arranged in a trabecular or glandular pattern. Hematoxylin and eosin (HE). Figure 3. Pancreas; horse. Islet-cell tumor with a well-developed fibrous capsule, trabecular or glandular arrangement of neoplastic cells, and focal Zellballen formation. HE. Figure 4. Pancreas; horse. Islet-cell tumor with glandular arrangement of neoplastic cells. The tumor cells are uniform, with a lightly eosinophilic to amphophilic granular cytoplasm, round to oval nuclei, and inconspicuous nucleoli. HE. Figure 5. Pancreas; horse. Islet-cell tumor, with moderate, diffuse, cytoplasmic immunoreactivity of neoplastic cells for glucagon; hyperplasia of alpha cells in islets (left). Immunohistochemistry, anti-glucagon antibody, hematoxylin counterstain. Figure 6. Pancreas; horse. Islet-cell tumor, with moderate, diffuse, cytoplasmic immunoreactivity of neoplastic cells for synaptophysin. Immunohistochemistry, antisynaptophysin antibody, hematoxylin counterstain. Figure 7. Pancreas; horse. Islet-cell tumor, with no immunoreactivity of neoplastic cells for insulin and a physiologically sized islet with strong, cytoplasmic staining of beta cells. Immunohistochemistry anti-insulin antibody; hematoxylin counterstain. 


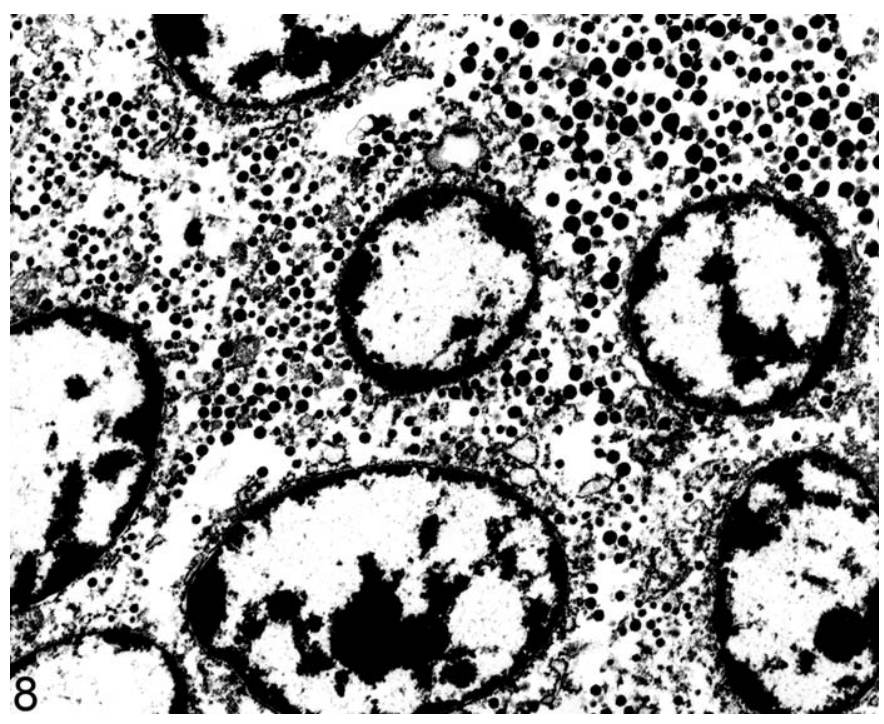

Figure 8. Pancreas; horse. Islet-cell tumor with numerous electrondense granules measuring 230 to $400 \mathrm{~nm}$ in diameter, similar to those observed in normal pancreatic alpha cells. Transmission electron microscopy.

Pan-NEN was diagnosed due to the lack of clinical signs associated with glucagonoma syndrome, such as diabetes mellitus and skin lesions. Serum chemistry data were not available, but hyperglycemia due to hyperglucagonemia was considered unlikely in this horse.

Nonfunctioning Pan-NENs are generally detected during the life of an animal due to signs produced by tumor mass effect or on postmortem examination as incidental findings. ${ }^{2,5}$ Since nonfunctioning Pan-NENs do not tend to produce hormonebased clinical syndromes, the incidence of these tumors in domestic animals is unknown. Most cases diagnosed in vivo are malignant and produce clinical disease secondary to widespread metastasis. ${ }^{5}$ Multiple malignant, most likely nonfunctional insulin-producing Pan-NENs have been reported in cattle. Interestingly, all cattle with multiple tumors also exhibited unilateral pheochromocytoma, as we observed in this case. ${ }^{4}$ A jaguar exhibiting 2 glucagon-producing Pan-NETs without clinical syndrome and no evidence for malignancy was described, ${ }^{12}$ and ferrets also have exhibited multiple Pan-NENs, mainly insulinomas. ${ }^{5}$ Horses, to the best of our knowledge, have not been diagnosed with any type of Pan-NEN so far. ${ }^{11}$ The pancreata of 165 horses of different age groups were investigated at the Institute of Veterinary Pathology, LMU Munich between 2009 and 2012. No other primary pancreatic tumors or diseases were observed by macroscopic investigation of the whole organ or by histological examination of 5 samples from different randomly chosen regions of the pancreas.

The pancreatic neoplasms of this case showed a predominantly glandular or trabecular growth pattern. Pan-NETs of humans and animals may show various arrangements of neoplastic cells, including solid, trabecular, glandular, pseudoacinar, and gyriform. ${ }^{1,2}$ Interestingly, a predominantly glandular arrangement of neoplastic cells has been explicitly described only in humans and dogs so far. ${ }^{2,9}$

In humans, $60 \%$ of all Pan-NETs are nonfunctioning. The tumors are usually 5 to $6 \mathrm{~cm}$ in diameter and frequently malignant, but due to advanced imaging techniques, smaller, nonfunctioning tumors are being diagnosed with higher frequency today. ${ }^{7}$ Pan-NENs that are classified as nonfunctional in veterinary medicine usually produce PP, glucagon, or somatostatin (ie, islet hormones that are thought to be less powerful in producing a clinical syndrome than insulin or gastrin). ${ }^{5}$ In humans, multiple tumors arising from microadenomas that are smaller than $0.5 \mathrm{~cm}$ are rare and usually associated with multiple endocrine neoplasia 1 (MEN1) or von Hippel-Lindau syndrome (VHL). Recently, multiple glucagon-producing tumors, developing from microadenomas (so-called glucagon-cell adenomatosis), were observed in 4 patients that were not associated with MEN1 or VHL. ${ }^{7}$ The tumors were randomly distributed throughout the pancreas, and patients exhibited up to 8 macrotumors and up to 600 microtumors. The tumor cells almost exclusively expressed glucagon, and an increased fraction of large islets also exhibited a reversed glucagon-to-insulin ratio, ${ }^{10}$ similar to the case presented here. In contrast, in islet-cell hyperplasia of domestic mammals, a normal distribution of the 4 main endocrine cells is retained. ${ }^{5}$ In 1 human patient exhibiting glucagon-cell adenomatosis, a homozygous missense mutation was found in the glucagon receptor gene that lowered the receptor's affinity to the ligand and reduced signal transduction, thereby possibly causing alpha-cell hyperplasia. An autosomal recessive mode of inheritance of the mutation was suggested. ${ }^{10}$ In MEN1 and VHL, endocrine-cell hyperplasia and microadenomas are considered precursor lesions of Pan-NETs. It was also suggested that sporadic endocrine hyperplasia, dysplasia, and microadenoma may give rise to sporadic Pan-NETs, ${ }^{10}$ which could also be speculated in the present case.

In summary, this is the first report of multiple glucagonproducing pancreatic neuroendocrine tumors or nonfunctional alpha-cell adenomas of a horse, presumably arising from multifocal alpha-cell hyperplasia.

\section{Acknowledgements}

We thank Lisa Pichl and Karin Stingl for excellent technical assistance.

\section{Declaration of Conflicting Interests}

The author(s) declared no potential conflicts of interest with respect to the research, authorship, and/or publication of this article.

\section{Funding}

The author(s) received no financial support for the research, authorship, and/or publication of this article.

\section{References}

1. Charles JA. Pancreas. In: Maxie MG, ed. Jubb, Kennedy and Palmer's Pathology of Domestic Animals. Edinburgh, UK: Saunders; 2007:389-424. 
2. Heitz PU, Komminoth P, Perren A, et al. Tumours of the endocrine pancreas: introduction. In: DeLellis AR, Lloyd RV, Heitz PU, et al, eds. WHO Classification of Tumours of Endocrine Organs. 4th ed. Lyon, France: IARC Press; 2004:177-182.

3. Jabbour SA, Davidovici BB, Wolf R. Rare syndromes. Clin Dermatol. 2006;24(4):299-316.

4. Kelley LC, Harmon BG, McCaskey PC. A retrospective study of pancreatic tumors in slaughter cattle. Vet Pathol. 1996; 33(4):398-406.

5. Kiupel M, Capen C, Miller M, et al. Histological Classification of Tumors of the Endocrine System of Domestic Animals. Washington, DC: Armed Forces Institute of Pathology; 2008.

6. Klöppel G. Tumour biology and histopathology of neuroendocrine tumours. Best Pract Res Clin Endocrinol Metab. 2007; 21(1):15-31.
7. Klöppel G. Classification and pathology of gastroenteropancreatic neuroendocrine neoplasms. Endocr Relat Cancer. 2011; 18(suppl 1):S1-S16.

8. Lurye JC, Behrend EN. Endocrine tumors. Vet Clin North Am Small Anim Pract. 2001;31(5):1083-1100.

9. O'Brien TD, Hayden DW, O'Leary TP, et al. Canine pancreatic endocrine tumors: immunohistochemical analysis of hormone content and amyloid. Vet Pathol. 1987;24(4):308-314.

10. Ouyang D, Dhall D, Yu R. Pathologic pancreatic endocrine cell hyperplasia. World J Gastroenterol. 2011;17(2):137-143.

11. Priester WA. Pancreatic islet cell tumors in domestic animals: data from 11 colleges of veterinary medicine in the United States and Canada. J Natl Cancer Inst. 1974;53(1):227-229.

12. Ramos-Vara JA, Miller MA, Preziosi D. Glucagonoma in a jaguar (Panthera onca). J Zoo Wildl Med. 2000;31(4):563-565. 